\title{
Suprimento de micronutrientes, adequação energética e progressão da dieta enteral em adultos hospitalizados
}

\author{
Adequacy of energy and micronutrient supply \\ and progression of enteral diet in \\ hospitalized adult patients
}

\author{
Vivian Cristine LUFT $^{1}$ \\ Diego de Matos VIEIRA' \\ Mariur Gomes BEGHETTO ${ }^{1,2}$ \\ Carísi Anne POLANCZYK ${ }^{1}$ \\ Elza Daniel de MELLO
}

\section{RE S U M O}

\section{Objetivo}

Avaliar a adequação da dieta enteral, em termos de micronutrientes e energia e identificar fatores interferentes na progressão da dieta enteral prescrita a adultos hospitalizados em um hospital geral de alta complexidade.

\section{Métodos}

Entre junho de 2004 e maio de 2005, adultos internados em um hospital de alta complexidade do sul do Brasil foram avaliados quanto às suas características clínicas e da prescrição da nutrição enteral. As características da nutrição enteral foram avaliadas e comparadas às recomendações diárias de ingestão, obtendo-se o percentual de adequação de nutrientes prescritos na dieta enteral em relação aos valores de recomendação para cada paciente. Os fatores associados à prescrição de energia foram identificados por meio de Regressão Linear Múltipla.

\section{Resultados}

Foram acompanhados 230 pacientes em uso de nutrição enteral. As recomendações diárias foram alcançadas satisfatoriamente para vitaminas hidrossolúveis (exceto ácido fólico), lipossolúveis (exceto vitamina D) e minerais (exceto cálcio). Em média, as prescrições iniciais de nutrição enteral ofereceram 24,0kcal $/ \mathrm{kg} / \mathrm{dia}$ (desvio-padrão=10,8, e valores mínimo e máximo de 4,3 a 69,2), e progrediram até 28,4kcal/kg/dia

1 Universidade Federal do Rio Grande do Sul, Programa de Pós-Graduação em Epidemiologia. R. Ramiro Barcelos, 2350, Sala 635, 90035-903, Porto Alegre, RS, Brasil. Correspondência para/Correspondence to: V.C.LUFT. E-mail: <vivi_luft@yahoo.com.br>.

2 Hospital de Clínicas de Porto Alegre, Comissão de Suporte Nutricional. Porto Alegre, RS, Brasil. 
(desvio-padrão=11,8, valores mínimo e máximo de 1,4 a 69,2). A recomendação de 25 a $35 \mathrm{kcal} / \mathrm{kg} / \mathrm{dia}$ foi prescrita para $32,6 \%$ dos pacientes. Para $15,7 \%$ dos pacientes foram prescritas acima de $40 \mathrm{kcal} / \mathrm{kg} / \mathrm{dia}$. Somente o índice de massa corporal e o número de dias de hospitalização, ajustados para a quantidade de energia já inicialmente prescrita, associaram-se de forma independente à prescrição energética final.

\section{Conclusão}

Pequena proporção das prescrições esteve adequada em relação à quantidade de energia, e a progressão da dieta enteral ocorreu independentemente das características clínicas dos pacientes.

Termos de indexação: Adulto. Dietoterapia. Nutrição enteral. Terapia nutricional.

\section{A B S T R A C T}

\section{Objective}

To asses the adequacy of enteral diet, in terms of micronutrients and energy and to identify factors that interfere in the progression of enteral diets prescribed to adults hospitalized in a high complexity general hospital.

\section{Methods}

From June 2004 to May 2005, adult patients hospitalized in a high complexity hospital, in Southern Brazil, were assessed in terms of enteral diet prescription and clinical characteristics. The characteristics of the enteral nutrition were assessed and compared with the recommended daily intakes, obtaining percentages of nutrient adequacy of the enteral diet. Factors associated with energy prescription were identified by multiple linear regression.

\section{Results}

Two hundred and thirty tube-fed patients were followed. The dietary reference intakes were satisfatory met for water-soluble vitamins (except for folic acid), fat-soluble vitamins (except for vitamin D) and minerals (except for calcium). The mean initial energy prescription was $24.0 \mathrm{kcal} / \mathrm{kg} / \mathrm{day}$ (standard deviation=10.8, minimum and maximum values ranging from 4.3 to $69.2 \mathrm{kcal} / \mathrm{kg} / \mathrm{day}$ ) and progressed to $28.4 \mathrm{kcal} / \mathrm{kg} / \mathrm{day}$ (standard deviation=11.8, minimum and maximum values ranging from 1.4 to $69.2 \mathrm{kcal} / \mathrm{kg} / \mathrm{day}$ ). The recommendation of 25 to $35 \mathrm{kcal} / \mathrm{kg} / \mathrm{day}$ was prescribed to $32.6 \%$ of patients. Forty or more $\mathrm{kcal} / \mathrm{kg} /$ day was presuibed to $15.7 \%$ of the patients. Only body mass index and the number of hospital stay days, adjusted to the energy prescribed at the beginning, were independently associated with the final energy prescription.

\section{Conclusion}

A small proportion of the prescriptions were adequate in terms of Kcal/kg/day, and the progression of enteral diet occurs regardless of the clinical characteristics of the patients.

Indexing terms: Adult. Diet therapy. Enteral nutrition. Nutrition therapy.

\section{N T R O D U ÇÃ O}

A desnutrição hospitalar é um problema freqüente em cerca de 50\% dos adultos hospitalizados ${ }^{1,2}$, com impacto sobre a morbimortalidade $^{3-6}$, o tempo de permanência ${ }^{1,3,7}$ e os custos hospitalares ${ }^{7}$. A nutrição enteral é a alternativa terapêutica que viabiliza a manutenção do estado nutricional em pacientes com impossibilidade parcial ou total de manter a via oral como rota de alimentação, devendo ser adotada como via de escolha, sempre que o trato gastrointestinal estiver funcionante ${ }^{8}$. A nutrição enteral, portanto, visa oferecer todos os nutrientes necessários para a manutenção da vida, o crescimento celular e tecidual, minimizando e/ou revertendo o impacto da desnutrição. Por se tratar de terapêutica de alta complexidade, não isenta de complicações, o planejamento e a implementação da nutrição enteral devem integrar o tratamento dos pacientes, especialmente nos indivíduos desnutridos ${ }^{9}$.

Diferentes métodos podem ser utilizados para estimar a necessidade energética dos indivíduos adultos ${ }^{10}$. Ainda assim, é comum que médicos e nutricionistas deparem-se com situações 
clínicas que dificultam a estimativa das necessidades nutricionais e escolha de fórmulas dietéticas apropriadas. A prescrição de 30 calorias por quilograma de peso corporal ao dia ( $\mathrm{kcal} / \mathrm{kg} / \mathrm{dia})^{11}$, variando entre 25 a $35 \mathrm{kcal} / \mathrm{kg} / \mathrm{dia}$, e de 0,8 a $1,2 \mathrm{~g} / \mathrm{kg} / \mathrm{dia}$ de proteínas ${ }^{12}$, é usualmente adotada no cuidado de pacientes hospitalizados. No entanto, naqueles severamente desnutridos a progressão da nutrição enteral deve ser mais lenta, a fim de evitar a síndrome de hiperalimentação ${ }^{13}$, não devendo o fornecimento de energia ultrapassar $40 \mathrm{kcal} / \mathrm{kg} / \mathrm{dia}$. A tolerância à nutrição enteral deve ser cuidadosamente monitorada. Distensão e dor abdominal, resíduo gástrico e vômitos limitam a progressão do suporte nutricional ${ }^{14-17}$. Além disso, o adequado suprimento de vitaminas e minerais, estabelecido a partir de estimativas derivadas de estudos de base populacional, deve ser assegurado ${ }^{18-22}$.

Ainda que a indicação, a prescrição e o acompanhamento criterioso dos pacientes submetidos à terapia nutricional enteral sejam regulamentados pelo Ministério da Saúde no Brasil ${ }^{23}$, não se dispõe de artigos que descrevam estimativas globais do uso de nutrição enteral em pacientes hospitalizados neste País. Neste sentido, este estudo tem por objetivo: a) avaliar a adequação das prescrições de nutrição enteral, do ponto de vista do fornecimento de energia para pacientes adultos hospitalizados, b) identificar os fatores associados à evolução da quantidade de energia oferecida durante a hospitalização (progressão da dieta), e c) verificar a contribuição da terapia nutricional enteral quanto ao suprimento apropriado de micronutrientes.

\section{MÉTODOS}

Entre junho de 2004 e maio de 2005, pacientes internados no Hospital de Clínicas de Porto Alegre, um hospital geral universitário de atenção terciária à saúde, foram prospectivamente avaliados quanto às suas características clínicas e à prescrição de nutrição enteral. Este estudo foi aprovado pela Comissão de Pesquisa e Ética em Saúde da instituição (protocolo $n^{\circ}$ 02-215).

Foram incluídos pacientes com idade igual ou superior a 18 anos, hospitalizados por motivos clínicos e/ou cirúrgicos, pelo Sistema Único de Saúde. Foram excluídos os pacientes internados no Centro de Terapia Intensiva (CTI) e na unidade de internação psiquiátrica, os com expectativa de hospitalização inferior a 72 horas, os com doença inflamatória intestinal, diarréia, portadores do vírus da imunodeficiência humana adquirida e os pacientes sem condições de ter seu peso corporal aferido, por impossibilitar a estimativa da necessidade energética diária em kcal/kg/dia. Os pacientes foram acompanhados até a alta hospitalar, ou até apresentarem algum dos critérios de exclusão.

Os pacientes foram identificados a partir de uma listagem informatizada, no momento da primeira prescrição de nutrição enteral, sendo, a seguir, avaliados quanto aos critérios de elegibilidade. Após inclusão, as variáveis acompanhadas foram: idade, especialidade médica, diagnóstico nutricional, número de dias de uso de nutrição enteral, tipo de sonda, fórmula(s) dietética(s) utilizada(s), modo de administração da nutrição enteral e presença de vômitos, resíduo gástrico, distensão e dor abdominal.

Todos os cuidados com os pacientes foram realizados pelas equipes assistentes, mantendose as rotinas institucionais estabelecidas, de modo que a equipe de pesquisa não interferiu em nenhum momento no atendimento individual dos pacientes. Assim, para todos os pacientes eleitos foi adotado o sistema aberto de nutrição enteral, seguindo procedimentos validados de produção, armazenamento, distribuição e administração, conforme legislação vigente ${ }^{23}$. Seguindo a rotina institucional, são adotados 5 padrões de dietas industrializadas, em pó ou líquidas, reconstituídas ou diretamente envasadas em frascos descartáveis em área física específica ${ }^{23}$ (dieta polimérica com fibras nas densidades energéticas de $1,0 \mathrm{kcal} / \mathrm{mL}$, $1,2 \mathrm{kcal} / \mathrm{mL}$ e 1,5kcal/mL; dieta polimérica sem fibras 1,2kcal/mL; e dieta oligomérica 1,2kcal/mL), além da possibilidade de modulação de dietas 
especiais. A dieta é fracionada em 6 a 8 frascos ao dia, distribuídos em intervalos regulares de 3 horas.

Todos os pacientes foram avaliados 3 vezes por semana, em dias intercalados, por investigadores treinados, utilizando-se o mesmo protocolo de pesquisa.

$\mathrm{Na}$ inclusão do estudo, os pacientes tiveram o estado nutricional avaliado pelo cálculo do índice de massa corporal (IMC), expresso em $\mathrm{kg} / \mathrm{m}^{2}$, pelo percentual de peso corporal em relação a seu peso usual e pela Avaliação Nutricional Subjetiva Global (ANSG)24.

Os micronutrientes prescritos na nutrição enteral foram avaliados pela diferença absoluta (em mg ou $\mu \mathrm{g}$ ) e percentual de adequação em relação aos valores das recomendações nutricionais (Dietary Reference Intakes - DRI), de acordo com idade e sexo. Para os micronutrientes abaixo foram adotadas as seguintes quantidades (Recommended Dietary Allowances - RDA): Vitamina C: homens: $75 \mathrm{mg}$, mulheres: $90 \mathrm{mg}$; Ácido fólico: 400 $\mu$ g; Ácido pantotênico: $5 \mathrm{mg}$; vitamina B12: 2,4 $\mu$; B6: até 50 anos: 1,3mg, $>$ 50anos, homens: 1,7mg, mulheres: 1,5mg; B3: homens: $16 \mathrm{mg}$, mulheres:14mg; B2: homens: 1,3mg, mulheres: 1,1mg; B1: homens: 1,2mg, mulheres: 1,1mg; E: 15mg; A: homens: $900 \mu \mathrm{g}$, mulheres: $700 \mu \mathrm{g}$; Zinco: homens: $11 \mathrm{mg}$, mulheres: 8mg; Ferro: homens: $8 \mathrm{mg}$, mulheres até 50anos: 18mg, >50anos: 8mg; Fósforo: $700 \mathrm{mg}$. Valores recomendados como ingestão adequada (Adequate Intakes - Al) foram adotados para: Biotina: $30 \mu \mathrm{g}$; K: homens: $120 \mu \mathrm{g}$, mulheres: $30 \mu \mathrm{g}$;

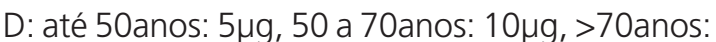
15 $\mathrm{gg}$; Fibra: 14g/1000kcal; Cálcio: até 50anos: $1000 \mathrm{mg}$, >50anos: $1200 \mathrm{mg}$ ). O suprimento das necessidades de micronutrientes foi considerado adequado quando atingiu valores iguais ou superiores a $100 \%$ das DRI ${ }^{18-22}$.

A prescrição da dieta também foi avaliada quanto ao fornecimento de energia e proteínas. A energia foi descrita em calorias por quilograma de peso corporal por dia ( $\mathrm{kcal} / \mathrm{kg} / \mathrm{dia})^{11}$, enquanto o fornecimento de proteínas foi expresso em gramas por quilograma de peso corporal por dia $(\mathrm{g} / \mathrm{kg} / \mathrm{dia})^{12}$. Foram consideradas adequadas, sob a perspectiva de fornecimento de energia, as prescrições que ofereceram entre 25 a $35 \mathrm{kcal} /$ $\mathrm{kg} / \mathrm{dia}$ (recomendado). Também foi descrito o percentual de prescrições acima de $40 \mathrm{kcal} / \mathrm{kg} / \mathrm{dia}$ (muito acima do recomendado). Os dados referem-se, exclusivamente, à dieta ofertada via sonda enteral, ainda que parte dos pacientes tenha recebido adicionalmente dieta por via oral e nutrição parenteral.

Foi calculada a diferença entre a quantidade de energia prescrita ao final e no início do uso da nutrição enteral. As médias da evolução (progressão) do fornecimento de energia foram comparadas pelo teste $t$ de Student, MannWhitney ou Kruskal Wallis. Para identificar os fatores que se associam à quantidade final de $\mathrm{kcal} / \mathrm{kg} /$ dia prescrita, foi realizada Regressão Linear Múltipla. A adequação do modelo multivariável foi avaliada quanto à sua forma funcional, à suposição de normalidade dos resíduos, à inexistência de autocorrelação e à homocedasticidade ${ }^{25}$.

Trata-se de uma amostra fixa de 230 pacientes. Neste sentido, considerando como clinicamente relevante uma diferença entre pacientes com ou sem fatores clínicos (como câncer e outras comorbidades, desnutrição, tipo de nutrição enteral e tolerância à nutrição enteral) de $5 \mathrm{kcal} / \mathrm{kg} / \mathrm{dia}$ na progressão da dieta, assumindo grande variabilidade (desvio-padrão foi de $7 \mathrm{kcal} / \mathrm{kg} / \mathrm{dia}$ ), para um valor $\alpha=0,05$, este estudo tem poder de $90 \%$ para identificar tal diferença para fatores presentes em $10 \%$ ou mais da população, e poder de aproximadamente $65 \%$ para detectar essa diferença para fatores presentes em $5 \%$ da população.

\section{RES U LT A D OS}

Foram acompanhados 230 pacientes com idade entre 20 e 99 anos (média - M=62 e desviopadrão - DP=15 anos), que fizeram uso de nutrição enteral por 1 a 57 dias (mediana, intervalo inter- 
quartil: 6, 10 - 18 dias). A média e o desvio-padrão do peso dos homens foram de 70,7 e $14 \mathrm{~kg}$, respectivamente, e das mulheres de 53,8 e 14,7kg, sendo o Índice de Massa Corporal médio de 21,6 ( $D P=4,7$, valores mínimo e máximo de 12,4 a 36,2) $\mathrm{kg} / \mathrm{m}^{2}$. A mediana (intervalo interquartil, IQ) do percentual de perda de peso em relação ao peso usual foi de $-12,8$ (IQ: -20,7 - -5,1), com valores mínimo e máximo de -50 a $+50 \%$. A mortalidade para esta população foi de $22,6 \%$ e o período médio de hospitalização foi de 23,8 ( $D P=17,9$, valores mínimo e máximo de 2 a 146) dias.

O fornecimento inicial de energia foi de $24(\mathrm{DP}=10,8$, valores mínimo e máximo de 4,3 a $69,2) \mathrm{kcal} / \mathrm{kg} / \mathrm{dia}$ e o final foi de 28,4 ( $\mathrm{DP}=11,8$, valores mínimo e máximo de 1,4 a 69,2) kcal/kg/

Tabela 1. Fornecimento energético inicial (em kcal/kg/dia), diferença do início ao fim da dieta enteral, e proporção de pacientes em uso de nutrição enteral com prescrição superior a 40kca/kg/dia, de acordo com características clínicas e do estado nutricional dos pacientes. Porto Alegre (RS), 2004 - 2005.

\begin{tabular}{|c|c|c|c|c|c|c|c|c|}
\hline \multirow[t]{2}{*}{ Características } & \multicolumn{3}{|c|}{ Fornecimento inicial de $\mathrm{kcal} / \mathrm{kg} / \mathrm{dia}$} & \multicolumn{3}{|c|}{$\begin{array}{l}\text { Evolução do fornecimento inicial de } \\
\mathrm{kcal} / \mathrm{kg} / \mathrm{dia} \text {, do inicial ao final }\end{array}$} & \multicolumn{2}{|c|}{$\begin{array}{l}\text { Prescrição >40 } \\
\text { kcal/kg/dia }\end{array}$} \\
\hline & Mediana & P25 - P75 & Valor $p$ & Média & $\left(I C_{95 \%}\right)$ & Valor $p$ & $\%$ & Valor $p$ \\
\hline $\operatorname{Sim}(n=161)$ & 20,7 & $15,4-27,7$ & $<0,01^{\dagger}$ & 4,7 & $3,5-5,9$ & 0,48 & 14 & 0,38 \\
\hline Não (n=69) & 23,9 & $19,4-33,7$ & & 3,9 & $2,3-5,6$ & & 19 & \\
\hline $\operatorname{sim}(n=5)$ & 16,4 & $10,3-33,8$ & $0,36^{+}$ & 6,2 & $-5,1-17,5$ & $0,80^{+}$ & 20 & $0,58^{\ddagger}$ \\
\hline Não $(n=225)$ & 21,7 & $16,7-29,5$ & & 4,4 & $3,4-5,4$ & & 15 & \\
\hline \multicolumn{9}{|l|}{ Diabetes } \\
\hline $\operatorname{sim}(n=21)$ & 22,6 & $18,8-28,0$ & $0,37^{\dagger}$ & 0,9 & $-2,0-3,5$ & $0,04^{+}$ & 9 & 0,39 \\
\hline \multicolumn{9}{|l|}{ Doença renal } \\
\hline $\operatorname{Sim}(n=8)$ & 18,8 & $14,9-22,2$ & $0,14^{+}$ & 1,2 & $-3,0-5,5$ & $0,25^{+}$ & 0 & $0,36^{\ddagger}$ \\
\hline Não $(n=222)$ & 22,0 & $16,6-28,5$ & & 4,6 & $3,6-5,6$ & & 16 & \\
\hline \multicolumn{9}{|l|}{$D P O C$} \\
\hline $\operatorname{Sim}(n=7)$ & 26,5 & $22,6-36,6$ & $0,08^{+}$ & 1,0 & $-1,2-3,2$ & $0,23^{\dagger}$ & 14 & $1,00 \neq$ \\
\hline Não $(n=223)$ & 21,5 & $16,4-29,3$ & & 4,6 & $3,5-5,6$ & & 16 & \\
\hline \multicolumn{9}{|l|}{ Insuficiência cardiaca } \\
\hline $\operatorname{Sim}(n=7)$ & 25,2 & $21,4-31,2$ & $0,29^{\dagger}$ & 1,8 & $-1,2-4,9$ & $0,19^{\dagger}$ & 14 & $1,00 \neq$ \\
\hline Não $(n=223)$ & 21,5 & $16,5-29,5$ & & 4,5 & $3,5-5,5$ & & 16 & \\
\hline Não $(n=59)$ & 16,1 & $12,4-21,3$ & & 5,4 & $2,1-4,5$ & & 3 & \\
\hline \multicolumn{9}{|l|}{ ANSG } \\
\hline Desnutrido grave $(n=137)$ & 23,0 & $19,2-31,3$ & $<0,01^{\S}$ & 5,5 & $3,6-6,5$ & $0,61^{\S}$ & 22 & $<0,01$ \\
\hline Desnutrido moderado $(n=53)$ & 21,5 & $15,0-29,0$ & & 3,4 & $1,4-5,2$ & & 9 & \\
\hline Nutrido $(n=40)$ & 16,6 & $13,0-20,9$ & & 3,9 & $2,4-5,4$ & & 2 & \\
\hline
\end{tabular}

DPOC: doença pulmonar obstrutiva crônica; ANSG: avaliação nutricional subjetiva global.

† Teste Mann-Whitney; ${ }^{\S}$ Teste Kruskal Wallis; ${ }^{\ddagger}$ Teste exato de Fisher; ${ }^{*} 23$ pacientes não tiveram sua altura aferida, impossibilitando o cálculo do IMC, ${ }^{* *} 12$ pacientes não puderam informar seu peso usual, impossibilitando o cálculo do percentual de perda de peso; \% proporção de pacientes. 
dia. O alvo energético, estimado em 25 a $35 \mathrm{kcal} /$ $\mathrm{kg} / \mathrm{dia}$, foi atingido por $21,7 \%$ dos pacientes ao início do uso da nutrição enteral e por 32,6\% ao final. Por outro lado, já no início do uso da dieta enteral mais de $40 \mathrm{kcal} / \mathrm{kg} / \mathrm{dia}$ foram prescritas a $9,1 \%$ dos pacientes e, ao final, a 15,7\%. O suprimento final de proteínas foi de $1,0(\mathrm{DP}=0,5$, valores mínimo e máximo de 0,1 a 3,1) g/kg/dia. Não foi observada diferença ao comparar a progressão de energia entre homens (4,9; IC95\%: $3,7-6,1 \mathrm{kcal} / \mathrm{kg})$ e mulheres $(3,7 ; \mathrm{IC} 95 \%: 2,0$ -
$5,4 \mathrm{kcal} / \mathrm{kg})(p=0,23)$ e entre pacientes clínicos $(4,1$; IC95\%: 2,8 - 5,5kcal/kg) e cirúrgicos (4,8; IC95\%: $3,3-6,2 \mathrm{kcal} / \mathrm{kg})(p=0,54)$.

Verificou-se que os pacientes diabéticos $(p=0,04)$, os com vômitos $(p=0,01)$ e os com resíduo gástrico $(p=0,06)$ tiveram menor progressão da dieta durante o período de uso da nutrição enteral (Tabelas 1 e 2). Ainda que sem significância do ponto de vista estatístico, pacientes com doença renal $(p=0,25)$, doença pulmonar obstrutiva crônica $(p=0,23)$, insuficiência cardíaca

Tabela 2. Fornecimento energético inicial (em kcal/kg/dia), diferença do início ao fim da dieta enteral, e proporção de pacientes em uso de nutrição enteral com prescrição superior a 40kcal/kg/dia, de acordo com o modo de administração da dieta e a tolerância à nutrição enteral. Porto Alegre (RS). 2004 - 2005.

\begin{tabular}{|c|c|c|c|c|c|c|c|c|}
\hline \multirow[t]{2}{*}{ Características } & \multicolumn{3}{|c|}{$\begin{array}{l}\text { Fornecimento inicial de } \\
\text { calorias } / \mathrm{kg} / \mathrm{dia}\end{array}$} & \multicolumn{3}{|c|}{$\begin{array}{l}\text { Evolução do fornecimento de } \\
\mathrm{kcal} / \mathrm{kg} / \text { dia, do inicial ao final }\end{array}$} & \multicolumn{2}{|c|}{$\begin{array}{c}\text { Prescrição }>40 \\
\mathrm{kcal} / \mathrm{kg} / \mathrm{dia}\end{array}$} \\
\hline & Mediana & $\mathrm{P} 25-\mathrm{P} 75$ & Valor $p$ & Média & $\left(\mathrm{IC}_{95 \%}\right)$ & Valor $p$ & $\%$ & Valor $p$ \\
\hline \multicolumn{9}{|c|}{ Tipo de sonda nutrição enteral } \\
\hline SNE $(n=213)$ & 22,0 & $16,8-29,4$ & $0,11^{\S}$ & 4,5 & $3,5-5,5$ & $0,52^{\S}$ & 15 & 0,21 \\
\hline Gastrostomia $(n=8)$ & 27,4 & $16,6-39,3$ & & 3,2 & $-9,5-15,9$ & & 37 & \\
\hline Jejunostomia $(n=9)$ & 16,1 & $8,0-29,0$ & & 4,1 & $-0,1-8,3$ & & 11 & \\
\hline \multicolumn{9}{|c|}{ Modo de administração da nutrição enteral } \\
\hline Intermitente $(n=183)$ & 21,4 & $16,7-29,1$ & $0,43^{\dagger}$ & 4,6 & $3,5-5,7$ & 0,63 & 15 & 0,46 \\
\hline Contínua $(n=47)$ & 23,1 & $15,3-35,1$ & & 4,0 & $1,8-6,1$ & & 19 & \\
\hline Gravidade $(n=215)$ & 22,0 & $16,9-29,5$ & $0,11^{\dagger}$ & 4,5 & $3,5-5,5$ & 0,82 & 15 & $0,71^{\neq}$ \\
\hline Bomba de infusão $(n=15)$ & 16,4 & $11,5-28,1$ & & 3,7 & $-3,6-11,0$ & & 20 & \\
\hline \multicolumn{9}{|l|}{ Uso da VO } \\
\hline $\operatorname{Sim}(n=126)$ & 20,8 & $16,5-26,1$ & $0,02^{+}$ & 4,4 & $3,0-5,8$ & 0,89 & 11 & 0,04 \\
\hline Não (n=104) & 23,0 & $16,7-33,9$ & & 4,5 & $3,1-5,9$ & & 21 & \\
\hline \multicolumn{9}{|l|}{ Uso de NP } \\
\hline $\operatorname{Sim}(n=5)$ & 12,4 & $8,0-21,5$ & $0,03^{+}$ & 1,9 & $-1,6-5,3$ & $0,26^{+}$ & 0 & $1,00^{\ddagger}$ \\
\hline Não (n=225) & 22,0 & $16,7-29,5$ & & 4,5 & $3,5-5,5$ & & 16 & \\
\hline \multicolumn{9}{|l|}{ Vômitos } \\
\hline $\operatorname{sim}(n=28)$ & 22,8 & $18,1-31,2$ & $0,25^{\dagger}$ & 2,0 & $0,2-3,8$ & 0,01 & 18 & $0,78^{\ddagger}$ \\
\hline Não $(n=202)$ & 21,5 & $16,5-29,5$ & & 4,8 & $3,7-5,9$ & & 15 & \\
\hline \multicolumn{9}{|l|}{ Resíduo gástrico } \\
\hline $\operatorname{Sim}(n=4)$ & 18,5 & $10,2-24,4$ & $0,27^{\dagger}$ & $-0,4$ & $-6,6-5,8$ & 0,06 & 0 & $1,00^{\ddagger}$ \\
\hline Não (n=226) & 21,8 & $16,5-29,5$ & & 4,5 & $3,5-5,5$ & & 16 & \\
\hline \multicolumn{9}{|l|}{ Distensão abdominal } \\
\hline $\operatorname{Sim}(n=42)$ & 21,0 & $15,3-28,1$ & $0,46^{\dagger}$ & 3,1 & $0,9-5,3$ & 0,20 & 14 & 0,78 \\
\hline Não $(n=188)$ & 21,6 & $16,8-29,8$ & & 4,8 & $3,6-5,9$ & & 16 & \\
\hline \multicolumn{9}{|l|}{ Dor abdominal } \\
\hline $\operatorname{Sim}(n=57)$ & 22,1 & $16,4-30,4$ & $0,62^{+}$ & 3,3 & $1,6-5,0$ & 0,18 & 16 & 0,97 \\
\hline Não (n=173) & 21,5 & $16,6-29,4$ & & 4,8 & $3,6-6,0$ & & 16 & \\
\hline
\end{tabular}

SNE: sonda nasoentérica; VO: possui via oral como rota auxiliar para nutrição; NP: possui via parenteral como rota auxiliar para nutrição.

${ }^{\dagger}$ Teste Mann-Whitney; ${ }^{\S}$ Teste Kruskal Wallis; ${ }^{\ddagger}$ Teste exato de Fisher. 
$(p=0,19)$, e em uso de nutrição parenteral $(p=0,26)$, também tiveram menor progressão da dieta enteral. Entretanto, aqueles com doença pulmonar obstrutiva crônica e insuficiência cardíaca já iniciaram o uso da nutrição enteral recebendo maior quantidade de $\mathrm{kcal} / \mathrm{kg} / \mathrm{dia}$.

Por meio da análise multivarida, verificouse que quanto maior o fornecimento inicial de energia $(p<0,01)$, quanto menor o IMC $(p<0,01)$ e maior o tempo de uso da nutrição enteral $(p<0,01)$, maior foi a prescrição final de energia, sendo esses três os fatores que se mantiveram independentemente associados à prescrição final da dieta. A Figura 1 (A) mostra uma tendência de relação inversa entre o número de $\mathrm{kcal} / \mathrm{kg} / \mathrm{dia}$ prescritas e o IMC do paciente, sendo observado maior fornecimento de energia aos pacientes desnutridos graves. Na Figura 1 (B) está apresentada a relação entre o número de dias de uso de nutrição enteral e a prescrição energética final atingida. Nela observa-se que não há um claro padrão de evolução na quantidade de kcal/kg/dia com o passar dos dias. Além disso, verifica-se que prescrições acima da recomendação ocorrem já na primeira semana de uso da nutrição enteral, e com maior faixa de variabilidade nos primeiros dias (Figura 1, B).

Quanto ao suprimento de micronutrientes (Tabela 3 e Figura 2), observa-se que as recomendações diárias de ingestão foram alcançadas satisfatoriamente para vitaminas hidrossolúveis (exceto ácido fólico), lipossolúveis (exceto vitamina D) e minerais (exceto cálcio). Foi identificado que o grupo de pacientes em que houve maior deficiência no suprimento de ferro foi o das mulheres de idade jovem, enquanto que, para vitamina $D$, o grupo dos idosos foi o que recebeu suprimento mais deficiente. O limite máximo de ingestão (Tolerable Upper Intake Levels - UL) foi ultrapassado para vitamina A $(>3000 \mu \mathrm{g} / \mathrm{dia}) \mathrm{em}$ 4 pacientes; para niacina (>35mg/dia), em 110 pacientes; para ácido fólico (>10000 $\mu \mathrm{g} / \mathrm{dia}), \mathrm{em}$ 2 pacientes; e para zinco ( $>40 \mathrm{mg} / \mathrm{dia})$, em 1 paciente.
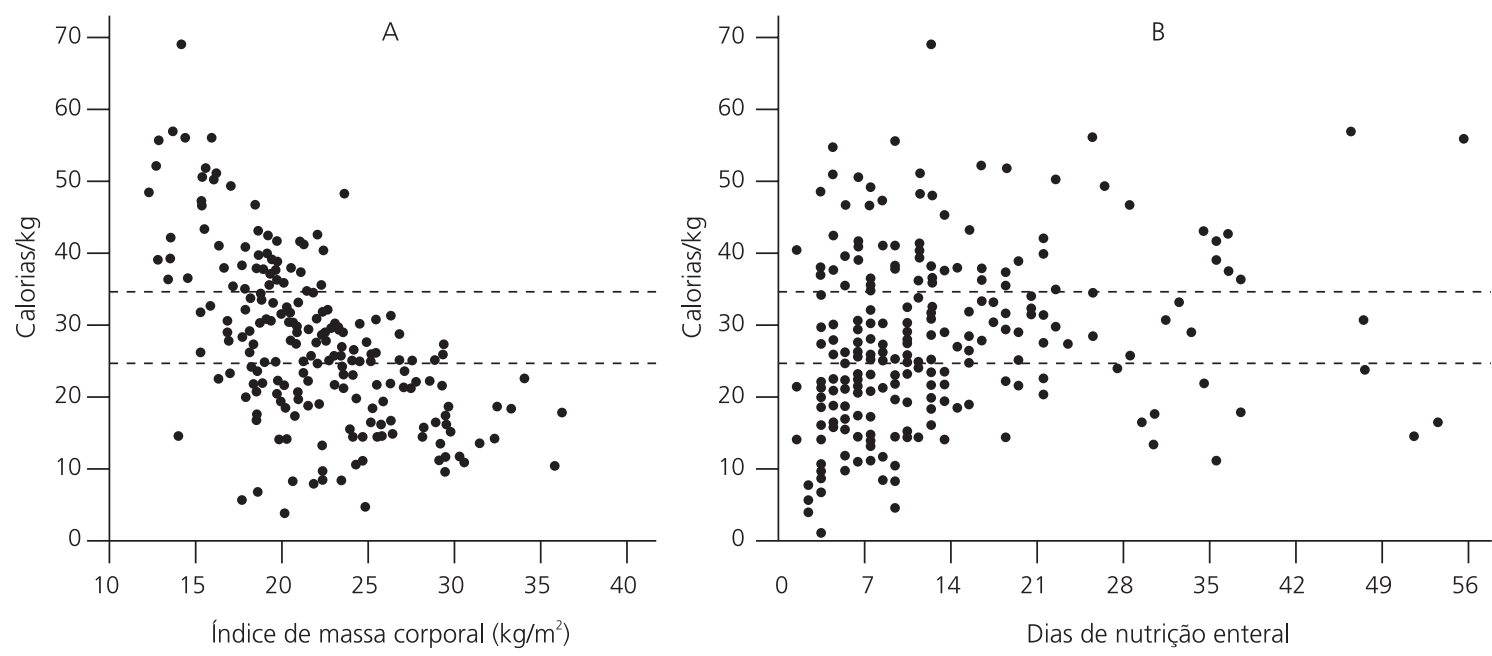

Figura 1. Relação entre o índice de massa corporal e o tempo de uso de nutrição enteral e o fornecimento final de energia, de pacientes em uso de nutrição enteral. Porto Alegre (RS), 2004-2005.

Nota: Salienta-se (entre as linhas tracejadas) o alvo da recomendação de 25 a 35 calorias/kg/dia. Cada ponto representa um indivíduo. 
520 | V.C. LUFT et al.

Tabela 3. Padrão de referência (Dietary Reference Intakes - DRI), quantidade de micronutrientes na dieta enteral prescrita, e a diferença entre o prescrito e o padrão de referência de pacientes em uso de nutrição enteral. Porto Alegre (RS), 2004 - 2005.

\begin{tabular}{|c|c|c|c|c|c|c|}
\hline \multirow[b]{2}{*}{ Vitamina C $(m g)^{\mathbf{b}}$} & \multirow[b]{2}{*}{$\begin{array}{l}\text { homens } \\
\text { mulheres }\end{array}$} & \multirow{2}{*}{$\begin{array}{c}\text { DRI } \\
75\end{array}$} & \multicolumn{2}{|c|}{ Prescrito $^{a}$} & \multicolumn{2}{|c|}{ Diferença $^{a}$} \\
\hline & & & $\begin{array}{l}210,0 \\
168,0\end{array}$ & $\begin{array}{r}(126,0-210,0) \\
(1,47-210,0)\end{array}$ & $\begin{array}{r}+135,0 \\
+78,0\end{array}$ & $\begin{array}{l}(+51,0-+135,0) \\
(+57,0-+120,0)\end{array}$ \\
\hline Ácido fólico $(\mu \mathrm{g})^{\mathbf{b}}$ & & 400 & 340,5 & $(252,0-420,0)$ & $-59,5$ & $(-148-+20,0)$ \\
\hline Ácido pantotênico $(\mathrm{mg})^{c}$ & & 5 & 12,9 & $(8,8-14,7)$ & $+7,9$ & $(+3,8-+9,7)$ \\
\hline Biotina $(\mu \mathrm{g})^{c}$ & & 30 & 191,1 & $(126,0-214,2)$ & $+161,1$ & $(+96,0-+184,2)$ \\
\hline Vitamina $B_{12}(\mu \mathrm{g})^{\mathbf{b}}$ & $<50$ anos & 2,4 & 5,7 & $(3,8-6,3)$ & $+3,3$ & $(+1,4-+3,9)$ \\
\hline Vitamina $B_{6}(m g)^{\mathbf{b}}$ & $\begin{array}{l}\geq 50 \text { anos, homens } \\
\geq 50 \text { anos, mulheres }\end{array}$ & $\begin{array}{l}1,3 \\
1,7\end{array}$ & $\begin{array}{l}3,4 \\
4,0\end{array}$ & $\begin{array}{l}(2,5-4,2) \\
(2,5-4,2)\end{array}$ & $\begin{array}{l}+2,1 \\
+2,3\end{array}$ & $\begin{array}{l}(+1,2-+2,9) \\
(+0,8-+2,5)\end{array}$ \\
\hline Vitamina $B_{3}(m g)^{\mathbf{b}}$ & $\begin{array}{l}\text { homens } \\
\text { mulheres }\end{array}$ & $\begin{array}{l}1,5 \\
16\end{array}$ & $\begin{array}{r}3,4 \\
38,6\end{array}$ & $\begin{array}{r}(2,8-4,2) \\
(25,2-42,0)\end{array}$ & $\begin{array}{r}+1,9 \\
+22,6\end{array}$ & $\begin{array}{r}(+1,3-+2,7) \\
(+9,2-+26,0)\end{array}$ \\
\hline Vitamina $B_{2}(m g)^{b}$ & $\begin{array}{l}\text { homens } \\
\text { mulheres }\end{array}$ & $\begin{array}{l}14 \\
1,3\end{array}$ & $\begin{array}{r}33,6 \\
4,2\end{array}$ & $\begin{array}{r}(25,2-42,0) \\
(2,8-4,6)\end{array}$ & $\begin{array}{r}+19,6 \\
+2,8\end{array}$ & $\begin{array}{r}(+11,2-+28,0) \\
(+1,5-+3,3)\end{array}$ \\
\hline Vitamina $B_{1}(m g)^{\mathbf{b}}$ & $\begin{array}{l}\text { homens } \\
\text { mulheres }\end{array}$ & $\begin{array}{l}1,1 \\
1,2\end{array}$ & $\begin{array}{l}3,7 \\
3,7\end{array}$ & $\begin{array}{l}(2,8-4,6) \\
(2,5-4,2)\end{array}$ & $\begin{array}{l}+2,6 \\
+2,6\end{array}$ & $\begin{array}{l}(+1,7-+3,5) \\
(+1,3-+3,0)\end{array}$ \\
\hline Vitamina $K(\mu \mathrm{g})^{c}$ & $\begin{array}{l}\text { homens } \\
\text { mulheres }\end{array}$ & $\begin{array}{l}1,1 \\
120\end{array}$ & $\begin{array}{r}3,4 \\
168,0\end{array}$ & $\begin{array}{r}(25,2-42,0) \\
(126,0-210,0)\end{array}$ & $\begin{array}{r}+2,3 \\
+48,0\end{array}$ & $\begin{array}{r}(+1,4-+3,1) \\
(+6,0-+90,0)\end{array}$ \\
\hline Vitamina $\mathrm{E}(\mathrm{mg})^{\mathbf{b}}$ & & $\begin{array}{l}90 \\
15\end{array}$ & $\begin{array}{r}168,0 \\
21,5\end{array}$ & $\begin{array}{r}(126,0-210,0) \\
(14,9-24,4)\end{array}$ & $\begin{array}{r}+78,0 \\
+6,5\end{array}$ & $\begin{array}{r}(+36,0-+120,0) \\
(0,0-+9,4)\end{array}$ \\
\hline Vitamina $D(\mu \mathrm{g})^{c}$ & $\begin{array}{l}<50 \text { anos } \\
50 \text { a } 70 \text { anos } \\
\geq 70 \text { anos }\end{array}$ & $\begin{array}{c}5 \\
10 \\
15\end{array}$ & $\begin{array}{l}8,4 \\
8,4 \\
9,4\end{array}$ & $\begin{array}{l}(6,3-10,5) \\
(7,9-10,5) \\
(7,9-10,5)\end{array}$ & $\begin{array}{r}+3,4 \\
-1,6 \\
-5,5\end{array}$ & $\begin{array}{r}(+1,3-+5,5) \\
(-3,7-+0,5) \\
(-7,0--4,5)\end{array}$ \\
\hline Vitamina A $(\mu \mathrm{g})^{\mathbf{b}}$ & $\begin{array}{l}\text { homens } \\
\text { mulheres }\end{array}$ & $\begin{array}{l}900 \\
700\end{array}$ & $\begin{array}{l}1495,4 \\
1329,2\end{array}$ & $\begin{array}{l}(996,9-1661,5) \\
(996,9-1661,5)\end{array}$ & $\begin{array}{l}+595,4 \\
+629,2\end{array}$ & $\begin{array}{r}(+96,9-+761,5) \\
(+296,9-+961,5)\end{array}$ \\
\hline Fibra $(g / 1000 \mathrm{kcal})^{c}$ & & 14 & 14,9 & $(14,9-14,9)$ & $+0,9$ & $(+0,9-+0,9)$ \\
\hline Zinco $(m g)^{\mathbf{b}}$ & $\begin{array}{l}\text { homens } \\
\text { mulheres }\end{array}$ & $\begin{array}{c}11 \\
8\end{array}$ & $\begin{array}{l}23,2 \\
20,2\end{array}$ & $\begin{array}{l}(15,1-25,2) \\
(15,3-25,2)\end{array}$ & $\begin{array}{l}+12,2 \\
+12,2\end{array}$ & $\begin{array}{l}(+4,1-+14,2) \\
(+7,3-+17,2)\end{array}$ \\
\hline Ferro $(m g)^{b}$ & $\begin{array}{l}\text { homens } \\
\text { mulheres, }<50 \text { anos } \\
\text { mulheres, } \geq 50 \text { anos }\end{array}$ & $\begin{array}{c}8 \\
18 \\
8\end{array}$ & $\begin{array}{l}16,8 \\
13,4 \\
13,4\end{array}$ & $\begin{array}{r}(10,1-18,0) \\
(9,7-20,2) \\
(10,6-16,8)\end{array}$ & $\begin{array}{r}+8,8 \\
-4,6 \\
+5,4\end{array}$ & $\begin{array}{r}(+2,01-+10,0) \\
(-8,2-+2,1) \\
(+2,6-+8,8)\end{array}$ \\
\hline Fósforo $(\mathrm{mg})^{\mathbf{b}}$ & & 70 & 924,0 & $(693,0-1155,0)$ & $+224,0$ & $(-7,0-+455,0)$ \\
\hline Cálcio $(\mathrm{mg})^{c}$ & $\begin{array}{l}<50 \text { anos } \\
\geq 50 \text { anos }\end{array}$ & $\begin{array}{l}1000 \\
1200\end{array}$ & $\begin{array}{l}1008,0 \\
1008,0\end{array}$ & $\begin{array}{l}(756,0-1260,0) \\
(756,0-1260,0)\end{array}$ & $\begin{array}{r}+8,0 \\
-192,0\end{array}$ & $\begin{array}{r}(-244,0-+260,0) \\
(-444,0-+60,0)\end{array}$ \\
\hline
\end{tabular}

a mediana (percentil 25 - percentil 75); b RDA: Recommended dietary allowances; ' Al: Adequate intaque.

Valores positivos indicam que o prescrito superou a recomendação; valores negativos indicam em quanto a prescrição não alcançou a recomendação.

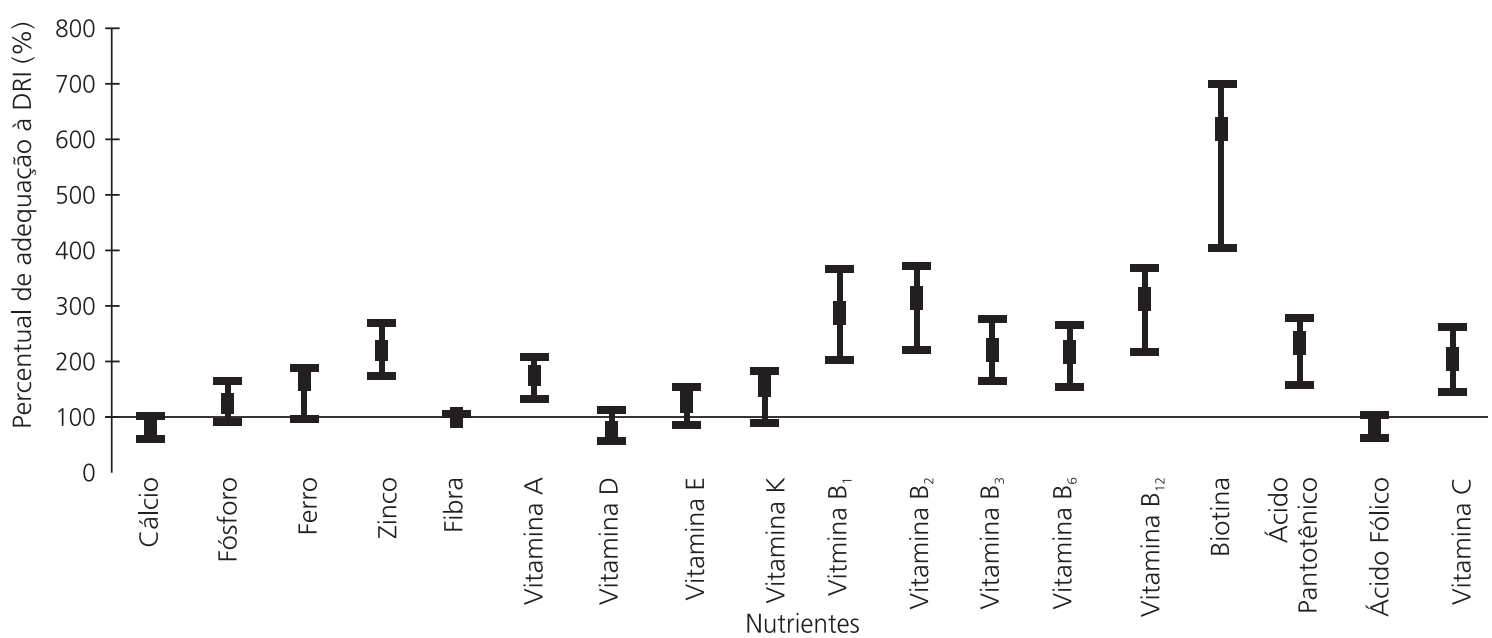

Figura 2. Mediana (percentil 25 - percentil 75) do percentual de adequação às recomendações dietéticas de ingestão (DRI) ${ }^{18-22}$ de pacientes em uso de nutrição enteral. Porto Alegre (RS), 2004-2005. 


\section{DIS CUSS Ã O}

A terapia nutricional enteral prescrita alcançou, para a maioria dos pacientes, as recomendações dietéticas vigentes para micronutrientes. É importante ressaltar que, apesar de os valores utilizados como referência terem sido derivados a partir de uma população saudável, constituem o melhor padrão de referência disponível. Sendo assim, sua aplicação em indivíduos hospitalizados ainda é a prática vigente. Futuramente, valores de referência específicos para condições de morbidade deverão ser estimados, de modo a permitir uma avaliação mais apropriada da adequação de micronutrientes no contexto hospitalar. No presente estudo, o suprimento de cálcio foi considerado o mais deficiente, sugerindo a necessidade de revisar, prioritariamente, a oferta deste nutriente nas dietas enterais adotadas como padrão nesta instituição.

Observou-se grande variabilidade no fornecimento de energia. A prescrição final de energia não esteve associada de forma significativa às características clínicas classicamente relacionadas à tolerância à dieta, sugerindo existir um padrão de prescrição que não valoriza estas informações. Mais do que isto, chama a atenção que, já no início do uso da nutrição enteral, pacientes tenham recebido prescrição de energia tão acima do recomendado.

Ainda que a nutrição enteral seja mais fisiológica que a nutrição parenteral ${ }^{8}$, sua administração requer vigilância, dadas as complicações metabólicas decorrentes da administração inadequada. Pouco foi descrito na literatura quanto à vigilância da dietoterapia no contexto hospitalar, com exceção à terapia intensiva. Em um estudo ${ }^{17}$ que avaliou a adequação da prescrição energética em indivíduos em ventilação mecânica alimentados por via enteral, foi verificado que para $68 \%$ dos pacientes foram prescritos valores abaixo do recomendado. No entanto, as necessidades energéticas dos pacientes foram estimadas pela equação de Harris \& Benedict ${ }^{26}$ que, como se sabe, superestima o gasto energético em cerca de
$500 \mathrm{kcal}$, quando comparado com o aferido pela calorimetria indireta (2 150 vs 1638 calorias/dia, respectivamente) ${ }^{17}$. Também raros são os estudos descrevendo fatores associados à progressão da nutrição enteral e o risco para hiperalimentação, ainda que o planejamento dietético e seu monitoramento sejam altamente recomendados ${ }^{12}$. Engel et al. ${ }^{15}$, utilizando calorimetria indireta, avaliaram a necessidade energética de 60 pacientes internados em uma unidade de tratamento intensivo. Os autores verificaram que a presença de sintomas gastrointestinais, intervenções cirúrgicas múltiplas, traumas abdominais, pélvicos e lombares comprometeram o fornecimento de energia na dieta.

Embora a associação entre uso de nutrição enteral e complicações metabólicas não possa ser estabelecida pelo presente estudo, o fornecimento excessivo de energia coloca os pacientes em risco para síndrome de hiperalimentação, caracterizada por hiperglicemia, hipertrigliceridemia, disfunção hepática, hipercapnia e azotemia, especialmente em pacientes criticamente doentes ${ }^{13,27}$. A elevada mortalidade observada neste estudo (22\%), bem como a longa permanência hospitalar (média de 24 dias), apontam para o fato de que usuários de sonda para alimentação enteral, ainda que não internados em um centro de terapia intensiva, constituem um grupo clinicamente comprometido e, conseqüentemente, suscetíveis a complicações relacionadas à evolução de sua doença e à própria terapêutica instituída, constituindo uma população de risco para hiperalimentação e suas complicações.

O diagnóstico de doença hepática, doença renal, doença pulmonar obstrutiva crônica e insuficiência cardíaca, a presença de resíduo gástrico, pouco parecem ter influenciado na prescrição final de energia, provavelmente por serem pouco freqüentes e representarem uma pequena parcela dos indivíduos neste estudo, comprometendo o poder estatístico. Por outro lado, o uso da via oral como rota assessória para nutrição também não teve influência sobre a quantidade de energia ofertada pela via enteral, 
embora o uso da via oral tenha sido freqüente (55\%). Este estudo apresenta somente a oferta de energia prescrita por meio de nutrição enteral, sem considerar o total energético prescrito por via oral e parenteral. Sendo assim, é razoável estimar que, se todas as vias de fornecimento de energia fossem consideradas, se encontraria oferta energética ainda maior e, conseqüentemente, maior inadequação em relação à recomendação.

Este estudo contribuiu para mostrar que, mesmo em um hospital de referência, onde os profissionais envolvidos com o acompanhamento nutricional desempenham importante papel na formação de futuros profissionais, há necessidade de revisar o processo de prescrição da nutrição enteral. Viu-se que prescrições não individualizadas, desconsiderando características clínicas, nutricionais e necessidades dietéticas, podem colocar os pacientes em risco de apresentarem complicações evitáveis. A adoção de mecanismos de vigilância clínica e epidemiológica pode assegurar que o melhor manejo nutricional seja instituído a pacientes hospitalizados em uso de nutrição enteral, considerando a oferta energética, protéica e de micronutrientes, nas rotinas hospitalares.

\section{A GRADECIMENTOS}

Ao Fundo de Incentivo à Pesquisa e Eventos (FIPE) do Hospital de Clínicas de Porto Alegre, pelo apoio à pesquisa, e à Coordenação de Aperfeiçoamento de Pessoal de Nível Superior (CAPES) que, por intermédio do Programa de Pós-Graduação em Epidemiologia da Universidade Federal do Rio Grande do Sul, concedeu uma bolsa de estudos a um dos pesquisadores.

\section{COLABORADORES}

V.C. LUFT, M.G. BEGHETTO, C.A. POLANCZYK e E.D. MELLO participaram na concepção e no desenho, na análise e na interpretação dos dados. D.M. VIEIRA na análise e interpretação dos dados.

\section{REFERÊ N CIAS}

1. Waitzberg DL, Caiaffa WT, Correia MI. Hospital malnutrition: the Brazilian national survey (IBRANUTRI): a study of 4000 patients. Nutrition. 2001; 17(7-8):573-80.

2. Mello ED, Beghetto MG, Teixeira LB, Luft VC. Desnutrição hospitalar: cinco anos após o IBRANUTRI. Rev Bras Nutr Clin. 2003; 18(2):65-9.

3. Naber TH, Schermer T, Bree A, Nusteling K, Eggink $\mathrm{L}$, Kruimel JW, et al. Prevalence of malnutrition in nonsurgical hospitalized patients and its association with disease complications. Am J Clin Nutr. 1997; 66(5):1232-9.

4. Heymsfield SB, Bethel RA, Ansley JD, Gibbs DM Felner JM, Nutter DO. Cardiac abnormalities in cachectic patients before and during nutritional repletion. Am Heart J. 1978; 95(5):584-94.

5. Arora NS, Rochester DF. Respiratory muscle strength and maximal voluntary ventilation in undernourished patients. Am Rev Respir Dis. 1982; 126(1):5-8

6. Anderson CF, Moxness K, Meister J, Burritt MF. The sensitivity and specificity of nutrition-related variables in relationship to the duration of hospital stay and the rate of complications. Mayo Clin Proc. 1984; 59(7):477-83.

7. Reilly JJ, Hull SF, Albert N, Waller A, Bringardener S. Economic impact of malnutrition: a model system for hospitalized patients. JPEN J Parenter Enteral Nutr. 1988; 12(4):371-6.

8. Peter JV, Moran JL, Phillips-Hughes J. A metaanalysis of treatment outcomes of early enteral versus early parenteral nutrition in hospitalized patients. Crit Care Med. 2005; 33(1): 213-20.

9. Brasil. Ministério da Saúde. Secretaria de Atenção à Saúde. Portaria n 31, de 8 de março de 2005. Define unidades de assistência de alta complexidade em terapia nutricional e centros de referência de alta complexidade em terapia nutricional e suas aptidões e qualidades. Diário Oficial da União. 200511 março.

10. Vinken $A G$, Bathalon GP, Sawaya AL, Dallal GE, Tucker KL, Roberts SB. Equations for predicting the energy requirements of healthy adults aged 18-81 y. Am J Clin Nutr. 1999; 69(5):920-6.

11. Stroud M, Duncan H, Nightingale J. Guidelines for enteral feeding in adult hospital patients. Gut. 2003; 52(Suppl 7):1-12

12. ASPEN Board of Directors and the Clinical Guidelines Task Force. Guidelines for the use of parenteral and enteral nutrition in adult and pediatric patients. JPEN J Parenter Enteral Nutr. 2002; 26(Suppl 1):1-138. 
13. Klein CJ, Stanek GS, Wiles CE. Overfeeding macronutrients to critically ill adults: metabolic complications. J Am Diet Assoc. 1998; 98(7): 795-806.

14. De Jonghe B, Appere-De-Vechi C, Fournier M, Tran B, Merrer J, Melchior JC, et al. A prospective survey of nutritional support practices in intensive care unit patients: what is prescribed? What is delivered? Crit Care Med. 2001; 29(1):8-12.

15. Engel JM, Muhling J, Junger $A$, Menges T, Karcher B, Hempelmann G. Enteral nutrition practice in a surgical intensive care unit: what proportion of energy expenditure is delivered enterally? Clin Nutr. 2003; 22(2):187-92.

16. Elpern EH, Stutz L, Peterson S, Gurka DP, Skipper A. Outcomes associated with enteral tube feedings in a medical intensive care unit. Am J Crit Care. 2004; 13(3):221-7.

17. O'Leary-Kelley CM, Puntillo KA, Barr J, Stotts N, Douglas MK. Nutritional adequacy in patients receiving mechanical ventilation who are fed enterally. Am J Crit Care. 2005; 14(3):222-31.

18. Institute of Medicine. Food and Nutrition Board: Dietary Reference Intakes: calcium, phosphorus, magnesium, vitamin D, and fluoride. Washington (DC): National Academy Press; 1997.

19. Institute of Medicine. Food and Nutrition Board. Dietary reference intakes: thiamin, riboflavin, niacin, vitamin $B_{6}$, folate, vitamin $B_{12}$, panthotenic acid, biotin, and choline. Washington (DC): National Academy Press; 1998.

20. Institute of Medicine. Food and Nutrition Board. Dietary reference intakes: vitamin C, vitamin $E$, selenium, and carotenoids. Washington (DC): National Academy Press; 2000.

21. Institute of Medicine. Food and Nutrition Board. Dietary reference intakes: vitamin A, vitamin K, arsenic, boron, chromium, copper, iodine, iron, manganese, molybdenum, nickel, silicon, vanadium, and zinc. Washington (DC): National Academy Press; 2001.

22. Institute of Medicine. Food and Nutrition Board. Dietary reference intakes: energy, carbohydrates, fiber, fat, fatty acids, cholesterol, protein, and amino acids. Washington (DC): National Academy Press; 2002.

23. Brasil. Ministério da Saúde. Secretaria de Vigilância Sanitária. Resolução RDC n 63, de 6 de julho de 2000. Aprova o Regulamento Técnico para fixar os requisitos mínimos exigidos para a Terapia de Nutrição Enteral. Diário Oficial da União. 2000.

24. Detsky AS, McLaughlin J, Baker JP, Johnston N, Whittaker S, Mendelson RA, et al. What is subjective global assessment of nutritional status? JPEN J Parenter Enteral Nutr. 1987; 11(1):8-13.

25. Kirkwood B, Sterne J. Essential medical statistics. 2nd ed. Oxford: Blackwell Science; 2003.

26. Harris JA, Benedict FG. A biometric study of basal metabolism in man. Washington (DC): Carnegie Institution of Washington; 1919.

27. Crook MA, Hally V, Panteli JV. The importance of the refeeding syndrome. Nutrition. 2001; 17(7-8): 632-7.

Recebido em: 16/2/2007

Versão final reapresentada em: 10/12/2007 Aprovado em: 2/6/2008 